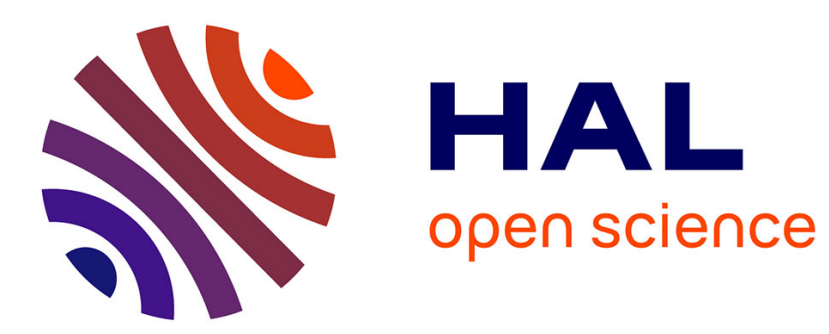

\title{
Facial Landmarks Localization Estimation by Cascaded Boosted Regression
}

\author{
Louis Chevallier, Jean-Ronan Vigouroux, Alix Goguey, Alexey Ozerov
}

\section{To cite this version:}

Louis Chevallier, Jean-Ronan Vigouroux, Alix Goguey, Alexey Ozerov. Facial Landmarks Localization Estimation by Cascaded Boosted Regression. International Conference on Computer Vision Theory and Applications (VISAPP 2013), Feb 2013, Barcelona, Spain. hal-00818808

\section{HAL Id: hal-00818808 \\ https://hal.inria.fr/hal-00818808}

Submitted on 29 Apr 2013

HAL is a multi-disciplinary open access archive for the deposit and dissemination of scientific research documents, whether they are published or not. The documents may come from teaching and research institutions in France or abroad, or from public or private research centers.
L'archive ouverte pluridisciplinaire HAL, est destinée au dépôt et à la diffusion de documents scientifiques de niveau recherche, publiés ou non, émanant des établissements d'enseignement et de recherche français ou étrangers, des laboratoires publics ou privés. 


\title{
Facial Landmarks Localization Estimation by Cascaded Boosted Regression
}

\author{
Louis Chevallier ${ }^{1}$, Jean-Ronan Vigouroux ${ }^{1}$, Alix Goguey ${ }^{1,2}$ and Alexey Ozerov ${ }^{1}$ \\ ${ }^{1}$ Technicolor, Cesson-Sévigné, France \\ \{louis.chevallier, jean-ronan.vigouroux, alexey.ozerov\}@technicolor.com \\ ${ }^{2}$ Ensimag, Saint-Martin d'Hères, France \\ alix.goguey@ensimag.imag.fr
}

Keywords: $\quad$ Face Landmarks Localization, Boosted Regression.

\begin{abstract}
Accurate detection of facial landmarks is very important for many applications like face recognition or analysis. In this paper we describe an efficient detector of facial landmarks based on a cascade of boosted regressors of arbitrary number of levels. We define as many regressors as landmarks and we train them separately. We describe how the training is conducted for the series of regressors by supplying training samples centered on the predictions of the previous levels. We employ gradient boosted regression and evaluate three different kinds of weak elementary regressors, each one based on Haar features: non parametric regressors, simple linear regressors and gradient boosted trees. We discuss trade-offs between the number of levels and the number of weak regressors for optimal detection speed. Experiments performed on three datasets suggest that our approach is competitive compared to state-of-the art systems regarding precision, speed as well as stability of the prediction on video streams.
\end{abstract}

\section{INTRODUCTION}

Facial landmarks detection is an important step in face analysis. Indeed, performance of face recognition or characterization systems (Everingham et al., 2006) greatly depends on the accuracy of this module. Accordingly, much work has been devoted to the problem of accurate and robust localization of facial landmarks. The importance of the required accuracy level depends on the final application. For example, for applications requiring a fine analysis of faces like lips-reading, a very precise localization of landmarks is needed. Typically, such high precision performance is required on near frontal faces; non-frontal positions are less likely to be subjected to these analyzes. Moreover, when this analysis involves motion video, temporal stability at a given precision rate is useful.

Most of state of the art landmark detectors (Cootes et al., 2001; Uřičář et al., 2012; Cao et al., 2012; Dantone et al., 2012; Vukadinovic and Pantic, 2005) are formulated as optimization or regression problems in some high-dimensional space (e.g., dozens of thousands of features). Thus, the precision of these approaches is limited by feature resolution. Using higher feature resolution (i.e., a feature space of much higher dimension), will in general not lead to improved precision due to limited training data, but instead entail over-fitting problems. We propose a new approach that allows increased feature resolution while keeping the feature space dimension unchanged, leading to higher landmark detection accuracy. This is achieved by using a cascade of boosted regressors, where the features used at each cascade level are extracted from a restricted area surrounding the corresponding landmark estimated by the previous levels of the cascade. We also discuss trade-offs between the number of cascades and the number of weak regressors for an optimal detection speed/precision ratio.

Our main contributions are:

1. A fast and accurate landmark position estimation algorithm, based on boosted weak regressors and Haar features extracted from the surrounding area, and a cascaded estimation scheme iterating on narrowing areas around each landmark.

2. A comprehensive assessment of the proposed estimator with regards to standard benchmarks, databases and state of the art landmark estimators, including an evaluation of its spatial stabil- 
ity. This is a new feature to our knowledge which is of great importance for the applications we are considering.

3. The flexibility of the proposed approach allows adjustment of the accuracy vs. computational load by simply varying the number of cascades.

The paper is organized as follows: related work and the proposed approach are described respectively in sections 2 and 3. Sections 4 and 5 are devoted to evaluation of performance and temporal stability. Some conclusions are drawn in section 6 .

\section{RELATED WORK}

The problem of predicting the location of facial landmarks consists in estimating the vector $S=$ $\left[x_{1}, y_{1}, \ldots x_{i}, y_{i}, x_{N}, y_{N}\right]^{T}$ comprising $N$ pairs of $2 \mathrm{D}$ coordinates based on the appearance of the face. To minimize ||$S-\hat{S} \|_{2}$, where $\hat{S}$ denotes an estimate, most of the existing approaches use optimization techniques (Cristinacce and Cootes, 2008; Uřičář et al., 2012) where the prediction is obtained as a solution of some optimization criterion, or regression techniques (Dantone et al., 2012; Valstar et al., 2010) where a function directly produces the prediction. Our approach follows the second direction.

Regarding data modeling, most approaches rely on both shape modeling representing a priori knowledge about landmarks locations and texture modeling corresponding to values of pixels surrounding the landmarks in the image itself, i.e., the posterior observations.

Active Shape Models (ASM) (Cootes et al., 1995) is a popular hybrid approach that uses a statistical model describing the shape (set of landmarks) of faces together with models of the appearance (texture) of landmarks. The prediction is iteratively updated to fit an example of the object in a new image. The shapes are constrained by the Point Distribution Model (PDM) (Kass et al., 1988) to vary only in ways seen in a training set of labeled examples. Active Appearance Models (AAM) (Cootes et al., 2001) are an extension of the ASM approach. In AAM, a global appearance model is used to optimize the shape parameters. Among the weaknesses frequently pointed out for this approach are the need for images of sufficiently high resolution, and the sensitivity to initialization. Our regression approach using Haar features computed over the face area, can, on the contrary, work with small images-in theory as small as the grid used for defining the set of Haar features-in our case $17 \times 17$, yielding 13920 features. Moreover, as a re- gression approach there is no iterative search process to be initialized.

A straightforward approach to landmark detection is based on using independently trained detectors for each facial landmark. For instance the AdaBoost based detectors and its modifications have been frequently used (Viola and Jones, 2001). If applied independently, the individual detectors often fail to provide a robust estimate of the landmark positions because of the weakness of local evidence. This can be solved by using a prior on the geometrical configuration of landmarks.

Valstar et al. (Valstar et al., 2010) proposed transforming the detection problem into a regression problem. They define a regression algorithm, based on Support Vector Regression, BoRMan, to estimate the positions of the feature points from Haar features computed at locations with maximum a priori probabilities. A Belief Propagation algorithm is used to improve the estimation of the target points, using a Markov Random Field modeling the relative positions of the points. Series of estimations are performed, by adding Gaussian noise to the current target estimation, and retaining the median of the predictions as the final estimation.

In (Everingham et al., 2006), a facial landmark detector is described which is based on the independent training of a local appearance model and the deformation cost of the Deformable Part Model-a structure which captures spatial relation between landmarks. The former relies on an AdaBoost classifier using Haar like features. The latter consists of a generative model using a mixture of Gaussian trees. In our evaluation section, we use an implementation of this system, which represents an optimization based solution to landmark detection.

Another approach based on regression for determining landmarks localization is described in (Cao et al., 2012). In this work, explicit multiple regression is used to directly predict landmarks localization. All landmarks coordinates (the shape) are predicted simultaneously by the regressor. The design relies on a cascaded structure: a top level boosted regressor uses weak regressors that are themselves boosted. These primary regressors use weak fern regressors: regression trees with a fixed number of leaves. In contrast with this system, our system consists of as many regressors as landmarks to be predicted. While (Cao et al., 2012) described a hierarchical structure, the structure of our system is a true cascade of regressors similar to the classifiers cascade proposed by (Viola and Jones, 2001) in their face detector. 


\section{LANDMARK POSITION ESTIMATION BY CASCADED BOOSTED REGRESSION}

We propose to estimate the position of the landmarks by using boosting and cascading techniques that lead to a fast and accurate result. The prediction of the coordinates $(x, y)$ of each landmark is done using a boosted regressor, based on Haar features computed on the detected face. A more precise localization is obtained using cascaded predictors. Each landmark is predicted independently of the others, instead of using a shape-based approach, as in (Lanitis et al., 1997; Cootes et al., 2001; Cristinacce and Cootes, 2008; Uřičář et al., 2012), and for each landmark the $x$ and $y$ coordinates are predicted independently. Actually, even if each landmark is predicted independently, a shape constraint is implicitly taken into account by the first regressor since the features used by this regressor are extracted from the totality of the face area. A final test could be made to detect and correct grossly erroneous landmarks. We believe that this approach is robust to partial occlusion, since variability of one landmark does not perturb the position of the others.

In contrast to (Valstar et al., 2010) we do not regress from different starting points, and take the median position as an estimator. We build a series of estimations of the positions of the landmarks, designed to converge to the sought landmark with high precision. At each step the regressor operates on increasingly narrow windows.

The image measurements used in our system are Haar features. This choice has the advantage that integral representations of images were readily available since they are typically required by the ubiquitous $\mathrm{Vi}$ ola and Jones face detector (Viola and Jones, 2001) we are using. The Haar features are defined based on a regular grid mapped on the shrinking image area to be analyzed. We set the size of the grid to $17 \times 17$ cells and we use eight Haar feature shapes (see figure 1). Scaling and translating them results in a total of 13,920 Haar features.
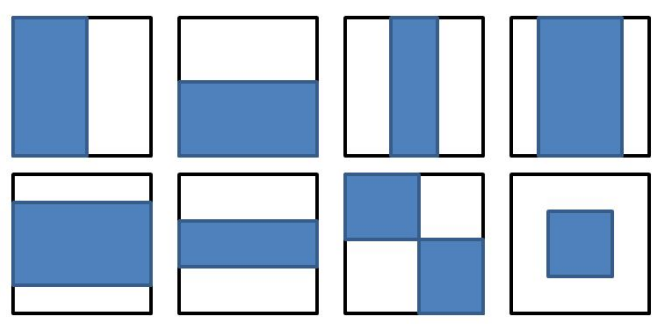

Figure 1: The eight Haar features used

\subsection{The First Level Regressor}

The first level regressor is a boosted regressor, using the algorithms described in (Friedman, 2001).

For the clarity of presentation we consider the case where we have only one coordinate of one landmark to predict for each image. Let $G$ be a set of $N$ images, and $y_{i}$ be the coordinate of the landmark on image $i$; the coordinates are measured in pixels from the top-left corner of the detection box. We have at our disposal a set of measurements (Haar features) on each image, used by the weak regressors and we want to create the most efficient strong predictor of the landmark coordinate, from a linear combination of weak predictors. Here the weak predictors consist of least-square fitted linear predictors using Haar features computed on the detected face (Viola and Jones, 2001).

The strong predictor is built iteratively as follows. Let $F$ be a matrix such that $F_{i j}$ is the value of the feature $F_{j}$ on image $i$. Let $Y^{(n)}$ be a vector containing the values to be predicted at iteration $n$. At first step $Y^{(1)}$ is initialized to the coordinates to predict: $Y^{(1)}=Y$, i.e., the vector of all the $y_{i}$. We predict $Y^{(n)}$ from $F_{j}$ using a standard linear predictor: $\widehat{Y^{(n)}}=a_{j} F_{j}+b_{j}$. The prediction error is $E_{j}^{(n)}=$ $Y^{(n)}-\widehat{Y^{(n)}}=Y^{(n)}-a_{j} F_{j}-b_{j}$, and the mean error is $e_{j}^{(n)}=\frac{1}{N}\left\|E_{j}^{(n)}\right\|$. The feature minimizing this error, $F_{j_{n}}$ is selected as $n^{t h}$ weak predictor. The predictions are subtracted from the value to predict, with a given weight $w_{n}$ set between 0.1 and 1 , and the new value to predict is thus:

$$
Y^{(n+1)}=Y^{(n)}-w_{n}\left(a_{j_{n}} F_{j_{n}}-b_{j_{n}}\right) .
$$

This is iterated $p$ times and results in a Linear strong predictor of the form:

$$
P^{(p)}(i)=\sum_{k=1}^{p} w_{k}\left(a_{j_{k}} F_{j_{k}}+b_{j_{k}}\right) .
$$

\subsection{Next Regression Levels}

The estimation of the position of a landmark can be improved by using the first estimation to re-center a window around the landmark of interest. This is the basis of our cascading process (see figure 2.)

The prediction window on the first level is the window detected by the face detector. In the second and subsequent levels it is a smaller window centered on the landmark position predicted by the previous level. For the size of the successive windows we use a decreasing ratio applied to the original face bounding box : 1.0, 0.8, 0.6, 0.4 for the four first levels. 
The levels of the cascade are therefore trained sequentially. The predictions of the previous level are used to train the next level.

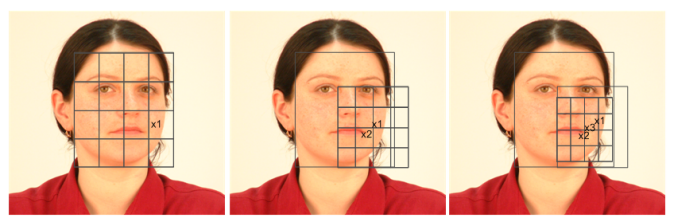

Figure 2: Three successive steps of regression for the Left Mouth Corner

\subsection{Other Weak Regressors}

As an alternative to Linear predictors for weak regressors we consider Non-Parametric weak-regressors. In this case, we bin the values of each feature $F_{j}$ and we estimate $y_{i}$ by the mean of $Y$ for the images falling in the same bin as $F_{i j}$. The boosting algorithm is applied as previously on the residual. This is somewhat equivalent to ferns (Dollár et al., 2010) using only one feature.

We have also experimented with gradient boosted trees (GBT) as weak regressors. Those regressors combining many Haar features were expected to provide more expressive power. Given the number of training image samples - ca. 5,000 - compared to the number of features $(13,920)$, the challenge was to prevent over-fitting. Optimal parameters were found through cross validation and we set the number of trees and maximal depth so that the total number of leaves (Haar features) were the same as in the two previous methods. In this experiment we are using 30 Haar features per weak regressor. The training time per landmark with 4 cascades is 8 minutes on an octocore $3 \mathrm{GHz}$ Intel processor.

We compare the merit of these three boosted weak regressors by testing them on the BioID database (Jesorsky et al., 2001) using four cascades (see table 1.)

Table 1: Percentage of tested images below error threshold on BioID dataset with three different weak regressors predicting the right of mouth corner.

\begin{tabular}{|l|c|c|c|}
\hline Percent. of tested images & $25 \%$ & $50 \%$ & $75 \%$ \\
\hline GBT & $2.2 \%$ & $3.4 \%$ & $5.0 \%$ \\
\hline Linear & $2.7 \%$ & $4.2 \%$ & $6.5 \%$ \\
\hline Non Parametric & $3.0 \%$ & $4.8 \%$ & $8.4 \%$ \\
\hline
\end{tabular}

We notice that GBT clearly outperforms the two other approaches.

\subsection{Parameters Settings}

Our approach uses two important parameters for training: the number of weak regressors and the number of cascade levels. In order to find the optimal choice we have tested several trade-offs presented in table 2. Of course the greater the computation ef-

Table 2: Error threshold at 50\% of tested images on BioID dataset with respect to weak regressors number and cascade levels.

\begin{tabular}{|c|c|c|c|c|}
\hline \# levels $\quad$ \# weaks & 15 & 30 & 45 & 60 \\
\hline 1 & 0.057 & 0.044 & 0.041 & 0.038 \\
\hline 2 & 0.047 & 0.039 & 0.036 & 0.034 \\
\hline 3 & 0.042 & 0.037 & 0.034 & 0.032 \\
\hline 4 & 0.040 & 0.037 & 0.033 & 0.032 \\
\hline
\end{tabular}

fort, the lower the error, but for a given computation load (i.e., the total number of Haar features which is proportional to the product numberOfWeaks $\times$ numberOfLevels), say 90, we can see that two levels and 45 weak regressors is the best choice.

\section{EVALUATION OF PERFORMANCE}

\subsection{Evaluation Methodology}

The models trained as described in the previous section were applied on three publicly available data sets with a manually labeled ground truth:

1. BioID (Jesorsky et al., 2001) is a very popular dataset containing 1,521 frontal face images with moderate variations in light condition and pose.

2. The PUT (Kasiński et al., 2008) dataset has 9,971 faces. The main source of face appearance variations comes from changes in poses and expressions .

3. The MUCT (Milborrow et al., 2010) dataset has 3,755 faces. It provides some diversity of lighting, age and ethnicity.

The set of landmarks provided by these databases are all different, so we retained a set of nine landmarks found on all three datasets and for which there is a good agreement regarding actual landmark positions: right and left corners of mouth, inner and outer corners of eyes, nose and nostrils (see figure 3.)

Our evaluation methodology consists of training our system with half of the images of each dataset 
and testing it on the rest. We use an evaluation metrics proposed in (Cootes et al., 2001) and defined as follows:

$$
m_{e}=\frac{1}{n s} \sum_{i=1}^{n} d_{i}
$$

where $d_{i}$ is the Euclidean distance between the ground truth landmark and the predicted one and $s$ is the interocular distance. $n=9$ is the number of landmarks.

\subsection{Results}

The systems against which we benchmarked our system are FLandmark (Uřičář et al., 2012), Oxford (Everingham et al., 2006), CLM (Cristinacce and Cootes, 2008), Kumar (Belhumeur et al., 2011), Valstar (Valstar et al., 2010) and Cao (Cao et al., 2012). The INRIA system is a variant of (Everingham et al., 2006) trained with a different training dataset.

We compare our system with recent systems for which the implementation was available ${ }^{1}$. For some others, we use the figures reported in corresponding papers on the same datasets: In the curves on figures 4

Table 3: Percentage of tested images below the average error threshold on BioID dataset.

\begin{tabular}{|l|c|c|c|}
\hline Percent. of tested images & $25 \%$ & $50 \%$ & $75 \%$ \\
\hline FLandmark & $5.4 \%$ & $5.5 \%$ & $7.0 \%$ \\
\hline Our system & $2 \%$ & $2.6 \%$ & $3.2 \%$ \\
\hline CLM & $2.5 \%$ & $4.5 \%$ & $6.5 \%$ \\
\hline Valstar & $1.5 \%$ & $3 \%$ & $5 \%$ \\
\hline
\end{tabular}

and 5 the error curve corresponds to the average error and to the maximum error observed on all the landmarks.

The obtained precision on PUT and MUCT images (figure 5) are not as good as on BioID (figure 4) because the pose of faces varies much more.

\section{EVALUATION OF TEMPORAL STABILITY}

\subsection{Motivation}

In practice, the accuracy of landmark prediction is limited by the modeling restriction, noise in the annotation and inherent ambiguity of the localization of facial landmark. $3 \%$ seems to be a performance that will be difficult to outperform.

\footnotetext{
${ }^{1}$ We did some tests with an implementation of (Valstar et al., 2010), but it gave results very different to what was reported in the paper, thus we do not present them.
}

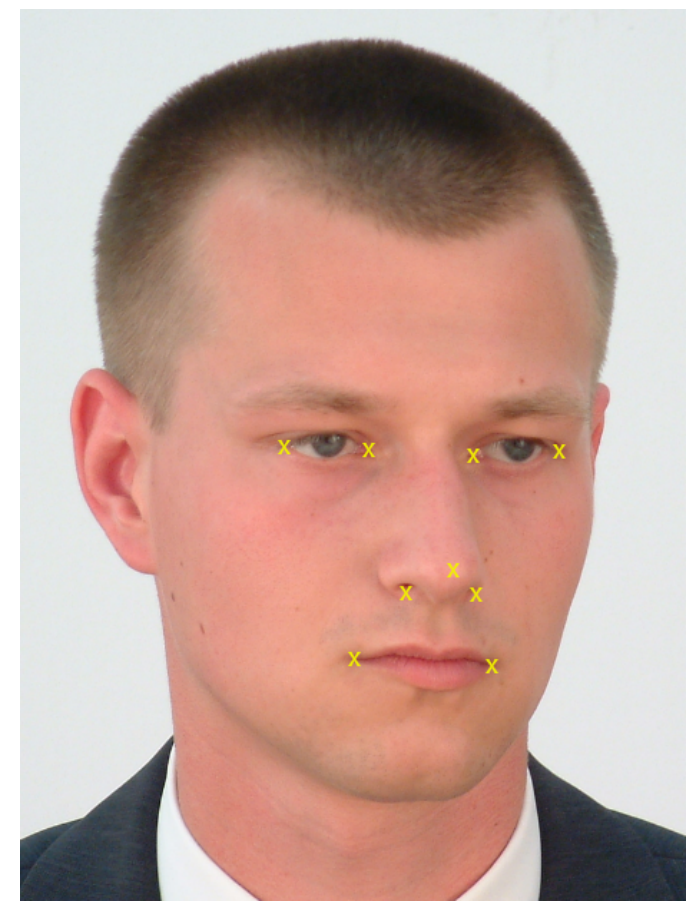

Figure 3: The nine landmarks used for the experiments.

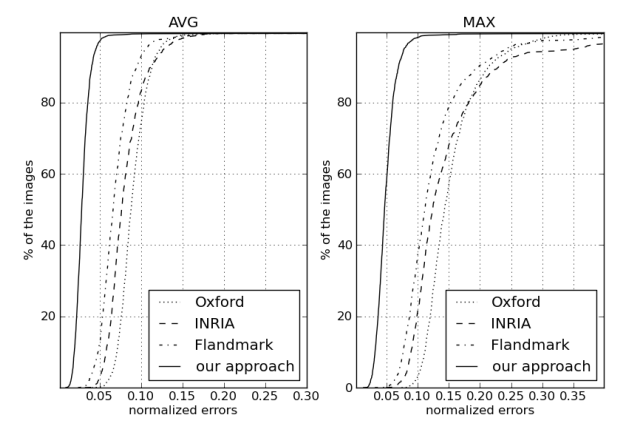

Figure 4: Cumulative distribution of point to point error measure on the BioID test set.

If a perfect accuracy cannot be reached, for some applications, it is important that the detector be as stable as possible. For example, the output of a landmark detector might be used as input of a speaking/nonspeaking classifier, which decides whether or not a visible face is currently speaking. Thus, if the landmarks are used to analyze the face (evolution of the mouth height or width), the noise due to the predictor should be kept as small as possible. If the accuracy is not good because of a constant bias, the prediction can still be useful.

Temporal stability of landmarks prediction has rarely been evaluated in the literature. We approach the problem by analyzing the normalized error over time. We propose to evaluate stability using auto- 

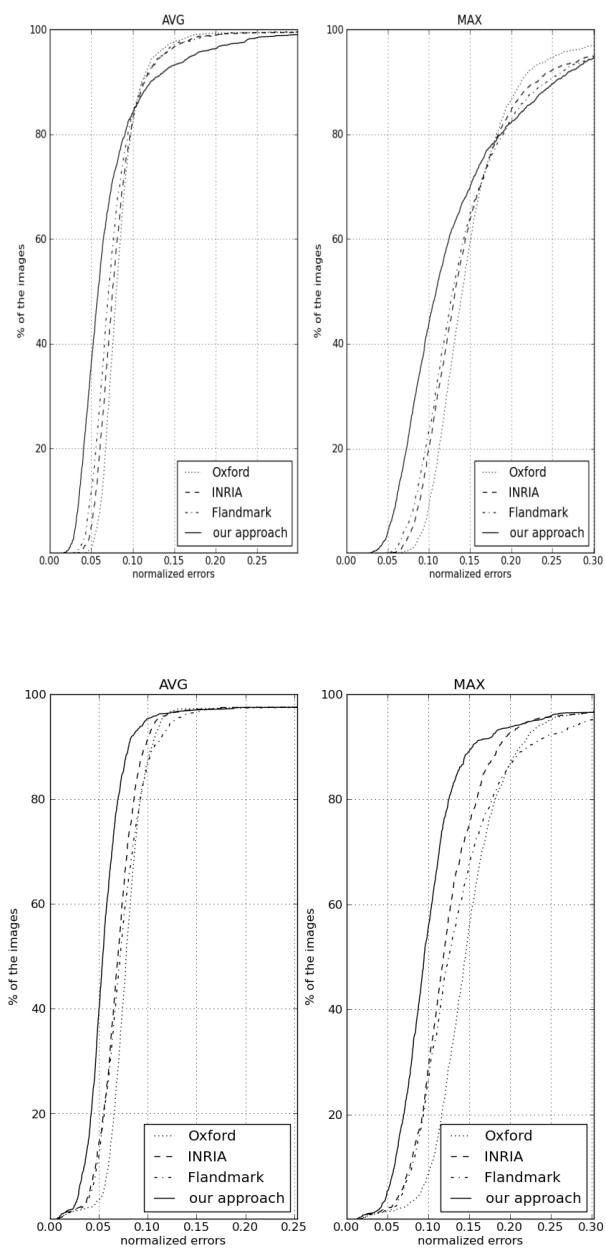

Figure 5: Cumulative distribution of point to point error measure on the PUT and MUCT test set.

correlation of the vectors of normalized errors corresponding to landmarks estimated for each frame of a video sequence. For this purpose, we have created our own ground truth of annotated frames. This dataset is comparable to the FGNET ${ }^{2}$ database but we found that we required more precision in the position of the landmarks than available in the latter for our comparison.

The auto-correlation vector ACor was calculated, using function Cor, as follows. $x$ and $y$ are two vectors and $N=\operatorname{Card}(x)=\operatorname{Card}(y)$, the cardinality of $x$ and $y . s$ is the shift index. Therefore, $A \operatorname{Cor}(x)=$ $\left(\operatorname{Cor}(x, x)_{s}\right)_{s \in \llbracket 1-N ; N-1 \rrbracket}$.

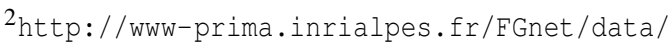
01-TalkingFace/talking_face.html
}

$$
\operatorname{Cor}(x, y)_{s}= \begin{cases}\operatorname{Cor}_{A}(x, y)_{s} & s \in A=[[0, N-1] \\ \operatorname{Cor}_{B}(x, y)_{s} & s \in B=[[1-N,-1]\end{cases}
$$

where

$$
\operatorname{Cor}_{A}(x, y)_{s}=\frac{\sum_{i=0}^{N-s}\left(x_{i+s}-\bar{x}_{s}\right)\left(y_{i}-\bar{y}_{s}\right)}{\sqrt{\sum_{i=0}^{N}\left(x_{i}-\bar{x}_{s}\right)^{2} \sum_{i=0}^{N}\left(y_{i}-\bar{y}_{s}\right)^{2}}}
$$

and

$$
\begin{aligned}
& \bar{x}_{s}=\frac{1}{2 * N-s} \sum_{i=0}^{N-s} x_{i+s} \\
& \bar{y}_{s}=\frac{1}{2 * N-s} \sum_{i=0}^{N-s} y_{i}
\end{aligned}
$$

Similarly:

$$
\operatorname{Cor}_{B}(x, y)_{s}=\operatorname{Cor}_{A}(y, x)_{-s}
$$

\subsection{Results}

In the two graphs represented in figure 6, we plot the auto-correlation of a constant error vector (as a baseline) and the normalized errors vector computed by three different detectors. The more stable a detector is, the closer to the baseline the corresponding curves should be.

We present here the results for two types of video streams: one with a speaker (whose head and lips are moving) and another with a quiet listener (who remains still). In each case, we show the graph corresponding to the left corner of mouth landmark error. The results show that our system has a better stability compared to the others in both types of video streams. The curve oscillations observed in the lower graph of figure 6 are due to a repetitive movement of the speaker lips. In the upper graph, the described feature does not have a repetitive pattern. This behavior can be regarded as an illustration of the superiority of regression techniques over optimization based techniques.

\section{CONCLUSION}

We have presented a technique of cascaded regression for direct prediction of facial landmarks. The algorithm consists of predicting successive 2D locations of the landmarks in a coarse to fine manner using a series of cascaded predictors, conferring robustness to the approach. Indeed predicting landmarks independently results in high precision since failure to find the good location of one of the landmarks does not propagate to the others. The regressors at each level of the 

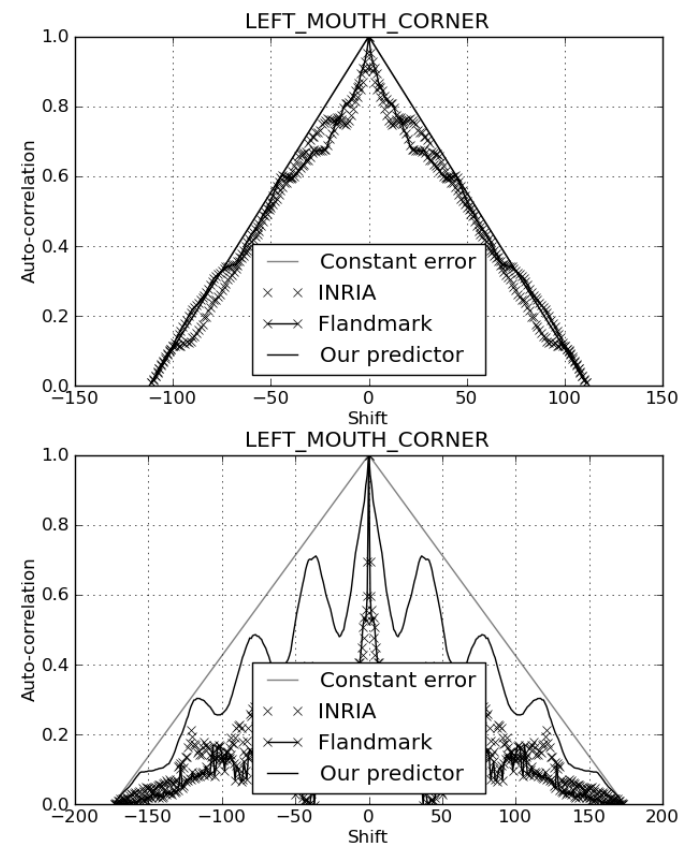

Figure 6: Auto-correlation of the normalized error vector of a non-speaking video sequence (upper graph) and a speaking one (lower graph).

cascade are based on gradient boosting. Three kinds of weak regressors have been assessed: linear regressors, non-parametric regressors and regression trees. The gradient boosted trees have the best performance. This simple scheme has proved to be very efficient compared to other tested approaches in terms of location errors. This approach is also very fast: it takes 8 milliseconds to compute the locations of 20 landmarks (not counting the computation of the integral image which is typically required for the detection of the face).

As possible extensions of the approach, we could consider applying a post-processing to the predicted landmarks by enforcing shape consistency (Belhumeur et al., 2011). An attractive capability of our model is to make it possible to trade precision against speed by traversing only a suitable number of levels of the cascade.

We believe that this generic approach could be applied to other problems involving regression where features derive from measurements from the signal e.g., to detection and localization of more generic objects using part based models.

\section{ACKNOWLEDGEMENTS}

This work was partially funded by the QUAERO project supported by OSEO and by the European integrated project AXES.

\section{REFERENCES}

Belhumeur, P. N., Jacobs, D. W., Kriegman, D. J., and Kumar, N. (2011). Localizing parts of faces using a consensus of exemplars. In The 24th IEEE Conference on Computer Vision and Pattern Recognition (CVPR).

Cao, X., Wei, Y., Wen, F., and Sun, J. (2012). Face alignement by explicit shape regression - to appear. In Proc. of CVPR'12.

Cootes, T. F., Edwards, G. J., and Taylor, C. J. (2001). Active appearance models. IEEE Transactions Pattern Analysis and Machine Intelligence, 23(6):681-685.

Cootes, T. F., Taylor, C. J., Cooper, D. H., and Graham, J. (1995). Active shape models - their training and application. Computer Vision and Image Understanding, 61(1):38-59.

Cristinacce, D. and Cootes, T. (2008). Automatic feature localisation with constrained local models. Pattern Recognition, 41(10):3054-3067.

Dantone, M., Gall, J., Fanelli, G., and Van Gool, L. (2012). Real-time facial feature detection using conditional regression forests. In Computer Vision and Pattern Recognition (CVPR).

Dollár, P., Welinder, P., and Perona, P. (2010). Cascaded pose regression. In Computer Vision and Pattern Recognition (CVPR), pages 1078-1085.

Everingham, M., Sivic, J., and Zisserman, A. (2006). Hello! my name is... Buffy - Automatic naming of characters in TV video. In Proceedings of the British Machine Vision Conference, volume 2.

Friedman, J. H. (2001). Greedy function approximation: A gradient boosting machine. Annals of Statistics, 29(5):1189-1232.

Jesorsky, O., Kirchberg, K. J., and Frischholz, R. (2001). Robust Face Detection using the Hausdorff distance. In $A V B P A$, pages $90-95$.

Kasiński, A., Florek, A., and Schmidt, A. (2008). The PUT face database. Image Processing and Communications, 13(3):59-64.

Kass, M., Witkin, A., and Terzopoulos, D. (1988). Snakes: Active contour models. International Journal of Computer Vision, 1(4):321-331.

Lanitis, A., Taylor, C. J., and Cootes, T. F. (1997). Automatic interpretation and coding of face images using flexible models. IEEE Transactions Pattern Analysis and Machine Intelligence, 19(7):743-756.

Milborrow, S., Morkel, J., and Nicolls, F. (2010). The MUCT Landmarked Face Database. Pattern Recognition Association of South Africa.

Uřičář, M., Franc, V., and Hlaváč, V. (2012). Detector of facial landmarks learned by the structured output svm. 
In Proceedings of the 7th International Conference on Computer Vision Theory and Applications. VISAPP '12.

Valstar, M., Martinez, B., Binefa, X., and Pantic, M. (2010). Facial point detection using boosted regression and graph models. In Proceedings of IEEE Int'l Conf. Computer Vision and Pattern Recognition (CVPR'10), pages 2729-2736, San Francisco, USA.

Viola, P. A. and Jones, M. J. (2001). Rapid object detection using a boosted cascade of simple features. In Computer Vision and Pattern Recognition (CVPR), pages 511-518.

Vukadinovic, D. and Pantic, M. (2005). Fully automatic facial feature point detection using gabor feature based boosted classifiers. In Proceedings of IEEE Int'l Conf. Systems, Man and Cybernetics (SMC'05), pages 1692-1698, Waikoloa, Hawaii. 